\title{
Mapping Risk from Genes to Behavior: The Enduring and Evolving Influence of Irving Gottesman's Endophenotype Concept
}

\author{
Danielle M. Dick \\ Departments of Psychology and Human \& Molecular Genetics, College Behavioral and Emotional Health Institute, Virginia \\ Commonwealth University, Richmond, VA, USA
}

\begin{abstract}
One of Irving I. Gottesman's many contributions to behavior genetics, and part of his enduring legacy, was his introduction of the term 'endophenotype' to the field of psychiatry. Gottesman argued that focusing on endophenotypes, rather than complex heterogeneous clinical diagnoses, could help elucidate disease etiology. Although a different strategy for gene identification ultimately proved successful (that of amassing extremely large sample sizes in order to overcome the 'noise' of heterogeneity and have sufficient power to find genes of very small effect), the endophenotype concept continues to make a meaningful contribution to the field. The endophenotype concept forced the field to move beyond a simple disease model of finding genes 'for' psychiatric outcomes, and reminded us that genes are quite distal from complex behavioral outcomes and disorders. Endophenotypes called our attention to the steps along that pathway. In that process, the concept of endophenotypes evolved and expanded to include discussion of the role that other intermediary traits and psychological processes play in the development and genetic etiology of psychiatric and substance use disorders. As large-scale consortia continues to identify genes and generate genomewide polygenic scores that are associated with behavioral outcomes, the next important step will be to characterize the pathways and mechanisms by which genetic risk unfolds. This essential step of mapping risk from genes to behavior is an evolution that follows naturally from the endophenotype concept, and could ultimately translate into improved prevention and intervention for individuals who are pre-disposed to mental health challenges.
\end{abstract}

Keywords: genetics, endophenotype, psychiatry

I was indoctrinated in the endophenotype concept early on. As an undergraduate student at the University of Virginia in the mid-1990s, I was fortunate to do my distinguished majors thesis under the supervision of Irving I. Gottesman, $\mathrm{PhD}$, a pioneer in the field of behavior genetics. He was fond of telling me how when he did his thesis on genetic influences on personality back in the late 1950s, the idea of genetic influence on behavior (gasp!) was considered outlandish and dismissed by leading researchers of the day. His seminar on schizophrenia led to my fascination with studying how genetic and environmental influences come together to contribute to complex behavioral disorders, an area that is still at the heart of my research program nearly 20 years later.

One of Irv's many contributions to behavior genetics, and part of his enduring legacy, was his introduction of the term 'endophenotype' to the field of psychiatry. In his 1972 book with James Shields (Gottesman \& Shields, 1972), they characterized endophenotypes as internal phenotypes discoverable by a 'biochemical test or microscopic examination', adapting the term from a 1966 paper addressing evolution and insect biology (John \& Lewis, 1966). The idea put forth was that focusing on endophenotypes, rather than complex heterogeneous clinical diagnoses, could help elucidate disease etiology. It was argued that broad binary classifications likely represented endpoints that were quite distal from the level of gene action; surely there was no gene 'for' schizophrenia, alcohol dependence, or any other complex behavior. Rather, genes affected biochemical processes and pathways that resulted in a downstream alteration in

RECEIVED 15 April 2018; ACCEPTED 30 April 2018

ADDress for correspondence: Danielle M. Dick, PhD, Department of Psychology and Human \& Molecular Genetics, College Behavioral and Emotional Health Institute, Virginia Commonwealth University, Box 842018, Richmond, VA 23284-2018, USA.E-mail: ddick@vcu.edu 
susceptibility to psychiatric disorder(s). So why not try to identify and study those 'endophenotypes' that were closer to the gene action? It was a brilliant idea.

But like many great ideas, it took a while to be recognized as such. The endophenotype concept did not gain widespread attention until it was reintroduced in his 2003 review paper (Gottesman \& Gould, 2003). The paper entered the field at a time of growing frustration surrounding failure to identify genes reliably associated with psychiatric disorders; endophenotypes represented a potential solution to the problem, with the idea that they should be 'simpler clues to genetic underpinnings than the disease syndrome itself' (Gottesman \& Gould, 2003, p. 636).

Finding these 'simpler' genetic components proved to be elusive. In a review of the endophenotype concept in psychiatry, Flint and Munafo (2007) challenged the idea that endophenotypes involved a simpler genetic architecture, with a review of the literature providing no support for the idea that the effect sizes of loci contributing to endophenotypes were any larger than those for genes contributing to psychiatric diseases themselves. This was true of psychiatric endophenotypes across a number of categories, including anatomical, developmental, electrophysiological, metabolic, sensory, and psychological/cognitive (Flint \& Munafo, 2007). A more recent review of electrophysiological endophenotypes provides continued evidence for the complex, polygenic nature of endophenotypes, which appear to have a genetic architecture very similar to that of observable behavioral traits (Iacono et al., 2017). In other words, there are many genes involved, and it is not easy to find them. Studying endophenotypes may have assisted with gene identification (Dick et al., 2006), but endophenotypes were certainly not the holy grail.

Ultimately, gene identification efforts for psychiatric disorders took a different direction. Rather than reducing etiological heterogeneity by focusing on more homogenous component processes, the field instead moved toward amassing tens or hundreds of thousands of affected individuals in order to overcome the 'noise' of heterogeneity and have sufficient power to find genes of very small effect (Schizophrenia Psychiatric Genome-Wide Association Study [GWAS] Consortium, 2011). This technique has proven to be successful (Clarke et al., 2017; Direk et al., 2017; Schizophrenia Working Group of the Psychiatric Genomics Consortium, 2014; Wood et al., 2014) - so, was the idea of endophenotypes misguided? Is it a concept that has outlived its usefulness? I would argue not.

Over the years, the endophenotype concept has sparked thoughtful intellectual exchanges about the very nature of psychiatric disorders and how to study their etiologies (Almasy \& Blangero, 2001; Cannon \& Keller, 2006; Lenzenweger, 2013). Several recent reviews have discussed the ways in which endophenotypes continue to be useful outside the context of gene identification, to include providing information about pathophysiological processes related to disease etiology, and how these processes may be shared across disorders (Beauchaine \& Constantino, 2017; Iacono, 2018; Miller \& Rockstroh, 2013). Indeed, the very concept of endophenotypes has evolved over the years and expanded beyond the original conceptualization as phenotypes discoverable by a 'biochemical test or microscopic examination' (Gottesman \& Shields, 1972, p. 319) and 'unseen by the unaided eye' (Gottesman \& Gould, 2003, p. 636). This expansion has included discussion of the role that other psychological traits and processes, such as personality, play in the development and genetic etiology of psychiatric and substance use disorders (Baker et al., 2005; Singh \& Waldman, 2005). Although this goes beyond how Gottesman and Shields (1972) originally intended the endophenotype concept to be used, I would argue that this may be one of its more long-lasting (if not immediately obvious) legacies.

The introduction of endophenotypes to the field of psychiatry forced movement beyond a simple disease model of finding genes for psychiatric outcomes. It reminded us that genes, while clearly important and influential, are quite distal from complex behavioral outcomes and disorders. There is a long, winding path from gene(s) to disorder, and endophenotypes forced our attention to the steps along that pathway. As large-scale consortia continues to identify genes and generate genome-wide polygenic scores that are associated with behavioral outcomes, we must be careful to remind ourselves repeatedly that those genetic variants are not genes for schizophrenia, depression, ADHD, and so forth. They are simply variants that are associated with the outcome, and those associations could arise through a variety of pathways and intermediate traits.

The next important step following the identification of genetic associations will be to characterize the pathways and mechanisms by which genetic risk unfolds (Dick et al., 2018). This will be critical in identifying targets for prevention and intervention in order to eventually ameliorate disease burden. I would argue that this essential step of mapping risk from genes to behavior is another evolution that follows from the endophenotype concept; it is the idea that we must study underlying mechanisms and steps in the long, winding path from genes to disorder. We must characterize the routes by which a particular gene (or genetic risk score) influences psychiatric outcome. These pathways will not simply include biochemical and physiological risk factors; the nature of genetic and environmental influences on psychiatric and substance use outcomes means that some of the risk is likely to unfold via temperamental and personality factors (Rothbart, 2011), and through environmental pathways (Scarr \& McCartney, 1983).

In the companion article in this issue 'Understanding Mechanisms of Genetic Risk for Adolescent Internalizing and Externalizing Problems: The Mediating Role of Parenting and Personality' (Su et al., this issue), we illustrate the important types of questions that can be examined 
following the identification of robust genetic risk scores, enabling us to understand how that risk unfolds across development to contribute to psychiatric outcomes. In a series of analyses, we characterized the risk associated with polygenic scores previously associated with major depressive disorder (Okbay et al., 2016). We found evidence that these scores were associated with both major depressive disorder symptoms and conduct disorder symptoms in adolescents. Importantly, these associations were mediated through personality factors and through parenting. Part of the 'genetic' risk for adolescent internalizing and externalizing disorders unfolded via reduced parental monitoring was evoked by the child's personality, contributing to their elevated risk for mental health challenges. These analyses illustrate the complex pathway by which genetic risk can impact disorder(s), suggesting that personality characteristics and evocative gene-environment correlation serve as two mechanisms by which genetic influences impact adolescent behavioral problems. It may not be the 'simpler' answer that Irv was seeking, but it certainly represents a step toward understanding the pathway(s) by which the genotype influences disorder.

Irv was fond of ending his talks with the statement, 'When the facts change, I change my mind. What do you do?' When it comes to the evolving nature, use, and characterization of endophenotypes, I think he would still be happy with the way the story has unfolded.

\section{Acknowledgment}

Dr Danielle M. Dick is supported by the National Institutes of Health/National Institute on Alcohol Abuse and Alcoholism (grant numbers R01 AA015416, K02 AA018755, P50 AA0022537, R01 AA026057, and U10 AA008401).

\section{Disclosure of Interests}

None.

\section{Details of Ethical Approval}

This article does not contain any studies with human participants or animals performed by the author.

\section{References}

Almasy, L., \& Blangero, J. (2001). Endophenotypes as quantitative risk factors for psychiatric disease: Rationale and study design. American Journal of Medical Genetics, 105, $42-44$.

Baker, L. A., Isen, J., Bezdjian, S., \& Raine, A. (2005). Are personality traits endophenotypes for antisocial behavior? Behavior Genetics, 35, 831-832.

Beauchaine, T. P., \& Constantino, J. N. (2017). Redefining the endophenotype concept to accommodate transdiagnostic vulnerabilities and etiological complexity. Biomarkers in Medicine, 11, 769-780.
Cannon, T. D., \& Keller, M. C. (2006). Endophenotypes in the genetic analyses of mental disorders. Annual Review of Clinical Psychology, 2, 267-290.

Clarke, T.-K., Adams, M. J., Davies, G., Howard, D. M., Hall, L. S., Padmanabhan, S., ... McIntosh, A. M. (2017). Genome-wide association study of alcohol consumption and genetic overlap with other health-related traits in UK Biobank $(N=112,117)$. Molecular Psychiatry, 22, $1376-$ 1384.

Dick, D. M., Barr, P. B., Cho, S. B., Cooke, M. E., Kuo, S. I. C., Lewis, T. J., ... \& Su, J. (2018). Post-GWAS in psychiatric genetics: A developmental perspective on the "other" next steps. Genes, Brain and Behavior, 17(3), e12447.

Dick, D. M., Jones, K., Saccone, N., Hinrichs, A., Wang, J. C., Goate, A., ... Hesselbrock, V. (2006). Endophenotypes successfully lead to gene identification: Results from the collaborative study on the genetics of alcoholism. Behavior Genetics, 36, 112-126.

Direk, N., Williams, S., Smith, J. A., Ripke, S., Air, T., Amare, A. T., ... Sullivan, P. F. (2017). An Analysis of two genomewide association meta-analyses identifies a new locus for broad depression phenotype. Biological Psychiatry, 82, 322329.

Flint, J. \& Munafo, M. R. (2007). The endophenotype concept in psychiatric genetics. Psychological Medicine, 37, 163180.

Gottesman, I. I., \& Gould, T. D. (2003). The endophenotype concept in psychiatry: Etymology and strategic intentions. American Journal of Psychiatry, 160, 636-645.

Gottesman, I. I., \& Shields, J. (1972). Schizophrenia and genetics: A twin study vantage point. London: Academic.

Iacono, W. G. (2018). Endophenotypes in psychiatric disease: Prospects and challenges. Genome Medicine, 10, 11.

Iacono, W. G., Malone, S. M., \& Vrieze, S. I. (2017). Endophenotype best practices. International Journal of Psychophysiology, 111, 115-144.

John, B., \& Lewis, K. R. (1966). Chromosome variability and geographical distribution in insects: Chromosome rather than gene variation provide the key to differences among populations. Science, 152, 711-721.

Lenzenweger, M. F. (2013). Thinking clearly about the endophenotype - Intermediate phenotype - Biomarker distinctions in developmental psychopathology research. Development and Psychopathology, 25, 1347-1357.

Miller, G. A., \& Rockstroh, B. (2013). Endophenotypes in psychopathology research: Where do we stand? Annual Review of Clinical Psychology, 9, 177-213.

Okbay, A., Baselmans, B. M., De Neve, J. E., Turley, P., Nivard, M. G., Fontana, M. A., ... Cesarini, D. (2016). Genetic variants associated with subjective well-being, depressive symptoms, and neuroticism identified through genomewide analyses. Nature Genetics, 48, 624-633.

Rothbart, M. K. (2011). Becoming who we are: Temperament and personality in development. New York, NY: Guilford Press. 
Scarr, S., \& McCartney, K. (1983). How people make their own environments: A theory of genotype greater than environment effects. Child Development, 54, 424-435.

Schizophrenia Psychiatric Genome-Wide Association Study (GWAS) Consortium. (2011). Genome-wide association study identifies five new schizophrenia loci. Nature Genetics, 43, 969-976.

Schizophrenia Working Group of the Psychiatric Genomics Consortium. (2014). Biological insights from 108 schizophrenia-associated genetic loci. Nature, 511, 421-427.

Singh, A. L., \& Waldman, I. (2005). Genetic and environmental influences on the covariation of negative emotionality and internalizing symptoms in children. Behavior Genetics, 35,832 .

Su, J., Kuo, S. I-Chun., Bucholz, K. K., Edenberg, H. J., Kramer, J. R., Schuckit, M., \& Dick, D. M. (this issue). Understanding mechanisms of genetic risk for adolescent internalizing and externalizing problems: The mediating role of parenting and personality. Twin Research and Human Genetics.

Wood, A. R., Esko, T., Yang, J., Vedantam, S., Pers, T. H., Gustafsson, S., ... Frayling, T. M. (2014). Defining the role of common variation in the genomic and biological architecture of adult human height. Nature Genetics, 46, 11731186. 